\title{
Correction to: Stickelberger ideals and Fitting ideals of class groups for abelian number fields
}

\author{
Masato Kurihara $^{1} \cdot$ Takashi Miura $^{2}$ \\ Published online: 5 June 2019 \\ (c) Springer-Verlag GmbH Germany, part of Springer Nature 2019
}

\section{Correction to: Math. Ann. (2011) 350:549-575 https://doi.org/10.1007/s00208-010-0570-y}

In the proofs of Theorems 3.5, 3.6 in [3], which give equality of the Fitting ideal of the class group of a CM-field and the Stickelberger ideal, we used Lemma 6.3 in [2], which bounds the index of the Fitting ideal in terms of the class number. But the proof of the lemma is not correct, more precisely, an exact sequence in the proof of Lemma 6.4 in [2] (on page 64 line 19), does not hold in general. We thank Takeshi Tsuji for giving us some comments on this.

In this correction, we give, without using Lemma 6.3 in [2], a proof of Theorems 3.5, 3.6 in [3]. Lemma 6.3 in [2] was also used in the proofs of Theorems 0.4, 0.6 in [2], so we also give in this article a correct proof of Theorems 0.4, 0.6 in [2]. Simply speaking, the proof in [3] (and in [2]) showing that the Fitting ideal is contained in the Stickelberger ideal is correct. Since Lemma 6.3 was used to show the other inclusion, our goal in this article is to give a correct argument for this inclusion.

For a discrete valuation ring $R$, we denote by $\operatorname{ord}_{R}$ the normalized additive valuation of $R$ such that $\operatorname{ord}_{R}(\pi)=1$ for a uniformizer $\pi$. For an $R$-module $M$, length ${ }_{R}(M)$ denotes the length of $M$ as an $R$-module. Let $K / k$ be an abelian extension as in $\S 3$ in [3]. Recall that we decomposed $\operatorname{Gal}(K / k)=\Delta_{K} \times \Gamma_{K}$ where \# $\Delta_{K}$ is prime to $p$, and $\Gamma_{K}$ is a $p$-group. For an arbitrary character $\psi$ of $\Gamma_{K}$ we denote by $K_{\psi}$ the fixed subfield of $K$ by $\operatorname{Ker} \psi$, and by $O_{\psi}$ the discrete valuation $\operatorname{ring} \mathbf{Z}_{p}$ [Image $\psi$ ] on which $\Gamma_{K}$ acts via $\psi$. Define $A^{\psi}:=\left(A_{K_{\psi}}\right)^{\psi}=A_{K_{\psi}} \otimes_{\mathbf{z}_{p}\left[\Gamma_{K}\right]} O_{\psi}$, which is an

The original article can be found online at https://doi.org/10.1007/s00208-010-0570-y.

$凶 \quad$ Masato Kurihara

kurihara@math.keio.ac.jp

Takashi Miura

t-miura@tsuruoka-nct.ac.jp

1 Department of Mathematics, Keio University, 3-14-1 Hiyoshi, Kohoku-ku, Yokohama 223-8522, Japan

2 Department of Creative Engennering, National Institute of Technology, Tsuruoka College, 104 Sawada, Inooka, Tsuruoka 997-8511, Japan 
$O_{\psi}\left[\Delta_{K}\right]$-module. We also use the notation $\psi$ for the ring homomorphisms induced by $\psi ; \psi: \mathbf{Z}_{p}[\operatorname{Gal}(K / k)] \rightarrow O_{\psi}\left[\Delta_{K}\right], \psi: \mathbf{Z}_{p}\left[\Gamma_{K}\right] \rightarrow O_{\psi}$, etc. For a character $\chi$ of $\Delta_{K}$ and $\mathbf{Z}_{p}[\mathrm{Gal}(K / k)]$-module $M$, we also use the notation $M^{\chi}$, defined by $M^{\chi}=M \otimes \mathbf{z}_{p}\left[\Delta_{K}\right] O_{\chi}$, which is now an $O_{\chi}\left[\Gamma_{K}\right]$-module. For a character $\chi$ of $\Delta_{K}$ and a character $\psi$ of $\Gamma_{K}$, we regard $\chi \psi$ as a character of $\operatorname{Gal}(K / k)$, and define $A^{\chi \psi}$ by $A^{\chi \psi}=A^{\psi} \otimes_{O_{\psi}\left[\Delta_{K}\right]} O_{\chi \psi}=\left(A_{K_{\psi}}\right)^{\psi} \otimes_{O_{\psi}\left[\Delta_{K}\right]} O_{\chi \psi}$, which is an $O_{\chi \psi}$-module. This module also coincides with $\left(A_{K_{\psi}}\right)^{\chi} \otimes_{O_{\chi}\left[\Gamma_{K}\right]} O_{\chi \psi}$.

We begin with the following proposition.

Proposition 1 We assume that an abelian extension $K / k$ satisfies the same conditions as that of Proposition 3.4 in [3]; namely, we assume that for every prime $\mathfrak{p}$ of $k$ above $p, \mathfrak{p}$ is unramified in $K$ and the ramification index of $\mathfrak{p}$ over $\mathbf{Q}$ is odd, and that the $\mu$-invariant of the cyclotomic $\mathbf{Z}_{p}$-extension of $K$ is zero, and that $K / k$ satisfies the condition $\left(\mathrm{A}_{p}\right)$ (The condition $\left(\mathrm{A}_{p}\right)$ means that $\Gamma_{K}$ is of the form $\Gamma_{K}=P_{\mathfrak{l}_{1}} \times \cdots \times P_{\mathfrak{l}_{r}}$ where $\mathfrak{l}_{i}$ 's are all ramifying primes of $k$ in $K / k$ and $P_{\mathfrak{l}_{i}}$ is the $p$-component of the inertia group of $\mathfrak{l}_{i}$ for each $\left.i\right)$. In addition, we assume that $K$ does not contain a primitive $p$-th root of unity. Let $\psi$ be a character of $\Gamma_{K}$ such that for every $i$ with $1 \leq i \leq r,\left.\psi\right|_{P_{\mathrm{l}_{i}}}$, the restriction of $\psi$ to $P_{\mathfrak{l}_{i}}$, is injective (faithful). Note that $K / K_{\psi}$ is an unramified extension.

For any $\psi \in \widehat{\Gamma}_{K}$ as above and any odd character $\chi \in \widehat{\Delta}_{K}$, we have

$$
\text { length }_{O_{\chi \psi}} A^{\chi \psi}=\operatorname{ord}_{O_{\chi \psi}}\left(L\left(0,(\chi \psi)^{-1}\right)\right) \text {. }
$$

In particular, we also have

$$
\psi\left(\operatorname{Fitt}_{O_{\chi}\left[\Gamma_{K}\right]}\left(A_{K_{\chi}}^{\chi}\right)\right)=\left(\psi\left(\theta_{K_{\chi} / k}^{\chi}\right)\right) .
$$

where $K_{\chi}$ is the fixed subfield of $K$ by Ker $\chi$.

Proof By Proposition 3.4 in [3], we have

$$
\text { Fitt }_{O_{\chi}\left[\Gamma_{K}\right]}\left(A_{K}^{\chi}\right) \subset\left(\Theta_{K / k} \otimes \mathbf{Z}_{p}\right)^{\chi}
$$

for all odd characters $\chi \in \widehat{\Delta}_{K}$. Since $\left[K: K_{\chi}\right]$ is prime to $p$, as we mentioned in the second paragraph of page 565 in [3], the usual norm argument shows that $A_{K}^{\chi} \simeq A_{K_{\chi}}^{\chi}$ and $\left(\Theta_{K / k} \otimes \mathbf{Z}_{p}\right)^{\chi}=\left(\Theta_{K_{\chi} / k} \otimes \mathbf{Z}_{p}\right)^{\chi}$. Furthermore, since $\Gamma_{K_{\chi}}=\Gamma_{K}$ and $K_{\chi} / K_{\chi \psi}$ is an unramified extension, we have $A_{K_{\chi}}^{\chi \psi} \simeq A_{K_{\chi \psi} \psi}^{\chi \psi}$ by Lemma 1.2 in [3] and $\left(\Theta_{K_{\chi} / k} \otimes \mathbf{Z}_{p}\right)^{\chi \psi}=\left(\theta_{K_{\chi \psi} \psi k}^{\chi \psi}\right)=\left(L\left(0,(\chi \psi)^{-1}\right)\right)$. Therefore, taking $\psi$ of (1), we have, for all odd characters $\chi \in \widehat{\Delta}_{K}$, Fitt $O_{\chi \psi}\left(A^{\chi \psi}\right) \subset\left(L\left(0,(\chi \psi)^{-1}\right)\right)$, equivalently

$$
\text { length }_{O_{\chi \psi}}\left(A^{\chi \psi}\right) \geq \operatorname{ord}_{O_{\chi \psi}}\left(L\left(0,(\chi \psi)^{-1}\right)\right) \text {. }
$$

Changing subscripts, we may assume that $P_{\mathfrak{l}_{1}} \simeq \mathbf{Z} / p^{n_{1}} \mathbf{Z}, \ldots, P_{\mathfrak{l}_{r}} \simeq \mathbf{Z} / p^{n_{r}} \mathbf{Z}$ and $n_{1} \geq \ldots \geq n_{r}$. We put $F=K_{\psi}$, and $K_{(\Delta)}=K^{\Gamma_{K}}$, the fixed subfield of $K$ by $\Gamma_{K}$. 
Since Image $\psi=\mu_{p^{n_{1}}} \subset \overline{\mathbf{Q}}_{p}^{\times}$, we have $\Gamma_{F}:=\operatorname{Gal}\left(F / K_{(\Delta)}\right) \simeq \mathbf{Z} / p^{n_{1}} \mathbf{Z}$. Let $\gamma$ be a generator of $\Gamma_{F}$ and $F_{1}$ the fixed subfield of $F$ by $\left\langle\gamma^{p^{n}-1}\right\rangle$, namely the unique subfield of $F / K_{(\Delta)}$ such that $\left[F: F_{1}\right]=p$. We will show that the following sequence is exact:

$$
0 \longrightarrow A_{F_{1}}^{\chi} \longrightarrow A_{F}^{\chi} \longrightarrow A_{F}^{\chi \psi} \longrightarrow 0
$$

In fact, since $F$ does not contain a primitive $p$-th root of unity by our assumption, for the unit group $E_{F}$ of $F$ we have $H^{1}\left(F / F_{1}, E_{F} \otimes \mathbf{Z}_{p}\right)^{-}=0$, which implies that the natural map $A_{F_{1}}^{-} \rightarrow A_{F}^{-}$is injective (cf. [1] Remark 2.2). Therefore, the map $A_{F_{1}}^{\chi} \rightarrow A_{F}^{\chi}$ is also injective. By the definition of $\psi$-quotient, we have $A_{F}^{\chi \psi} \simeq$ $A_{F}^{\chi} /\left(1+\gamma^{p^{n_{1}-1}}+\gamma^{2 p^{n_{1}-1}}+\cdots+\gamma^{(p-1) p^{n_{1}-1}}\right) A_{F}^{\chi}=A_{F}^{\chi} / N_{\operatorname{Gal}\left(F / F_{1}\right)} A_{F}^{\chi}$, which implies that the map $A_{F}^{\chi} \rightarrow A_{F}^{\chi \psi}$ is surjective. Since the norm map $A_{F}^{-} \rightarrow A_{F_{1}}^{-}$is surjective (cf. Lemma 1.2 in [3]), we have Image $\left(A_{F_{1}}^{\chi} \rightarrow A_{F}^{\chi}\right)=N_{\mathrm{Gal}\left(F / F_{1}\right)} A_{F}^{\chi}$. This shows the exactness at the middle term of the above sequence.

Since $A^{\chi \psi} \simeq A_{F}^{\chi \psi}$, the above exact sequence implies that

$$
\text { length }_{O_{\chi}}\left(A^{\chi \psi}\right)=\text { length }_{O_{\chi}}\left(A_{F}^{\chi}\right)-\text { length }_{O_{\chi}}\left(A_{F_{1}}^{\chi}\right) \text {. }
$$

For two characters $\chi_{1}, \chi_{2} \in \widehat{\Delta}_{K}$, we denote $\chi_{1} \sim \chi_{2}$ if these are $\mathbf{Q}_{p}$-conjugate (cf. page 555 in [3]). Until the end of the proof of this proposition, we use the following notation: $\sum_{\chi}$ (resp. $\sum_{\chi / \sim}$ ) means the sum which is taken over all odd characters of $\Delta_{K}$ (resp. taken over the equivalence classes of all odd characters of $\Delta_{K}$ ). We also use the notations $\bigoplus_{\chi}, \bigoplus_{\chi / \sim}$ in a similar way. We have the isomorphisms $A_{F}^{-} \simeq \bigoplus_{\chi / \sim} A_{F}^{\chi}$ and $A_{F_{1}}^{-} \simeq \bigoplus_{\chi / \sim} A_{F_{1}}^{\chi}$ because the order of $\Delta_{K}$ is prime to $p$. Put $f_{\chi}:=\left[\mathbf{Q}_{p}(\right.$ Image $\left.\chi): \mathbf{Q}_{p}\right]$ for each odd character $\chi \in \widehat{\Delta}_{K}$, and denote by $\operatorname{ord}_{p}$ the normalized valuation on $\overline{\mathbf{Q}}_{p}$ such that $\operatorname{ord}_{p}(p)=1$. By the analytic class number formula, we have

$$
\begin{aligned}
& \sum_{\chi / \sim} f_{\chi} \text { length }_{O_{\chi \psi}}\left(A^{\chi \psi}\right) \\
& =\sum_{\chi / \sim} f_{\chi} \text { length }_{O_{\chi}}\left(A^{\chi \psi}\right)=\sum_{\chi / \sim} f_{\chi}\left(\text { length }_{O_{\chi}}\left(A_{F}^{\chi}\right)-\operatorname{length}_{O_{\chi}}\left(A_{F_{1}}^{\chi}\right)\right) \\
& =\sum_{\chi / \sim}\left(\operatorname{ord}_{p}\left(\sharp A_{F}^{\chi}\right)-\operatorname{ord}_{p}\left(\sharp A_{F_{1}}^{\chi}\right)\right)=\operatorname{ord}_{p}\left(\sharp A_{F}^{-}\right)-\operatorname{ord}_{p}\left(\sharp A_{F_{1}}^{-}\right) \\
& =\sum_{\chi} \sum_{\substack{i=1 \\
(i, p)=1}}^{p^{n_{1}}} \operatorname{ord}_{p}\left(L\left(0,\left(\chi \psi^{i}\right)^{-1}\right)\right)=\sum_{\chi / \sim} f_{\chi}(p-1) p^{n_{1}-1} \operatorname{ord}_{p}\left(L\left(0,(\chi \psi)^{-1}\right)\right) \\
& =\sum_{\chi / \sim} f_{\chi} \operatorname{ord}_{O_{\chi \psi}}\left(L\left(0,(\chi \psi)^{-1}\right)\right) .
\end{aligned}
$$


This equality and the inequality (2) show that

$$
\text { length }_{O_{\chi \psi}}\left(A^{\chi \psi}\right)=\operatorname{ord}_{O_{\chi \psi}}\left(L\left(0,(\chi \psi)^{-1}\right)\right)
$$

for each odd character $\chi \in \widehat{\Delta}_{K}$.

Corollary 3.3 in [3]. In the proof of this corollary, Lemma 6.3 in [2] was not used, so the proof need not be changed. But it refers to Theorem 0.4 in [2] whose proof used Lemma 6.3 in [2]. So we give a correct proof of Theorem 0.4 here.

Proof of Theorem 0.4 in [2]. Let $K$ be an imaginary abelian number field such that no prime of $K^{+}$above $p$ splits in $K / K^{+}$. It is enough to show the equalities

$$
\operatorname{Fitt}_{O_{\chi}\left[\Gamma_{K}\right]}\left(A_{K}^{\chi}\right)=\left(\Theta_{K / \mathbf{Q}} \otimes \mathbf{Z}_{p}\right)^{\chi}
$$

for all odd characters $\chi \in \widehat{\Delta}_{K}$. As we mentioned in the proof of Proposition 1, we know that $A_{K}^{\chi} \simeq A_{K_{\chi}}^{\chi}$ and $\left(\Theta_{K / k} \otimes \mathbf{Z}_{p}\right)^{\chi}=\left(\Theta_{K_{\chi} / k} \otimes \mathbf{Z}_{p}\right)^{\chi}$. Therefore, the equality (3) is equivalent to the equality

$$
\operatorname{Fitt}_{O_{\chi}\left[\Gamma_{K}\right]}\left(A_{K_{\chi}}^{\chi}\right)=\left(\Theta_{K_{\chi} / \mathbf{Q}} \otimes \mathbf{Z}_{p}\right)^{\chi} .
$$

For $\chi \neq \omega$, by Corollary 0.10 in [2] and Lemma 3.2 in [3], we obtain the equality (4) using the same argument as in the paragraph following Lemma 3.2 in [3] (on page 562). For $\chi=\omega$, by Lemma 2.3 in [2], we may assume that $K_{\omega} / \mathbf{Q}$ satisfies the condition (A), and can apply Proposition 2.4 (2) in [3] to get the equality (4).

Proof of Theorem 3.6 in [3]. Let $K / k$ be an abelian extension satisfying the same conditions as that of Theorem 3.6 in [3], which are exactly the same as the conditions of Proposition 1 above. We have to prove that

$$
\text { Fitt }_{O_{\chi}\left[\Gamma_{K}\right]}\left(A_{K}^{\chi}\right)=\left(\Theta_{K / k} \otimes \mathbf{Z}_{p}\right)^{\chi}
$$

for all odd characters $\chi \in \widehat{\Delta}_{K}$. Each of these equalities is equivalent to the equality

$$
\operatorname{Fitt}_{O_{\chi}\left[\Gamma_{K}\right]}\left(A_{K_{\chi}}^{\chi}\right)=\left(\Theta_{K_{\chi} / k} \otimes \mathbf{Z}_{p}\right)^{\chi}
$$

as we mentioned above. By Proposition 3.4 in [3], we have

$$
\operatorname{Fitt}_{O_{\chi}\left[\Gamma_{K}\right]}\left(A_{K_{\chi}}^{\chi}\right) \subset\left(\Theta_{K_{\chi} / k} \otimes \mathbf{Z}_{p}\right)^{\chi}
$$

for all odd characters $\chi \in \widehat{\Delta}_{K}$ (Note that all $\chi$ 's are different from the Teichmüller character because $\mu_{p} \not \subset K$ by our assumption).

We show the other inclusion

$$
\left(\Theta_{K_{\chi} / k} \otimes \mathbf{Z}_{p}\right)^{\chi} \subset \operatorname{Fitt}_{O_{\chi}\left[\Gamma_{K}\right]}\left(A_{K_{\chi}}^{\chi}\right) .
$$


By the definition of Stickelberger ideal, we know easily that $\left(\Theta_{K_{\chi} / k} \otimes \mathbf{Z}_{p}\right)^{\chi}$ is generated by $\left\{v_{K_{\chi} / F}\left(\theta_{F / k}^{\chi}\right) \mid F \in \mathscr{M}_{K_{\chi} / k}\right\}$ (see page 47 in [2] for the definition of $\mathscr{M}_{K_{\chi} / k}$ ). Since $\left[K_{(\Delta)}: k\right]$ is prime to $p$, we know that $\theta_{K_{(\Delta)} / k}^{\chi} \in \operatorname{Fitt}_{O_{\chi}\left[\Gamma_{K_{(\Delta)}}\right]}\left(A_{K_{(\Delta)}}^{\chi}\right)$. By induction, we may assume that $\theta_{F / k}^{\chi} \in \operatorname{Fitt}_{O_{\chi}\left[\Gamma_{F}\right]}\left(A_{F}^{\chi}\right)$ for any $F \in \mathscr{M}_{K_{\chi} / k}, F \neq K_{\chi}$. We have to show that

$$
v_{K_{\chi} / F}\left(\theta_{F / k}^{\chi}\right) \in \operatorname{Fitt}_{O_{\chi}\left[\Gamma_{K}\right]}\left(A_{K_{\chi}}^{\chi}\right) .
$$

By the condition $\left(\mathrm{A}_{p}\right)$, we can reduce to the case that there is a prime $v$ of $k$ such that the only primes above $v$ are ramified in $K_{\chi} / F$. In this case, the inclusion $v_{K_{\chi} / F}\left(\operatorname{Fitt}_{O_{\chi}\left[\Gamma_{F}\right]}\left(A_{F}^{\chi}\right)\right) \subset \operatorname{Fitt}_{O_{\chi}\left[\Gamma_{K}\right]}\left(A_{K_{\chi}}^{\chi}\right)$ can be proved by the same argument as that on page 62 in [2]. This proves that $\nu_{K_{\chi} / F}\left(\theta_{F / k}^{\chi}\right) \in \operatorname{Fitt}_{O_{\chi}\left[\Gamma_{K}\right]}\left(A_{K_{\chi}}^{\chi}\right)$.

Thus it suffices to prove $\theta_{K_{\chi} / k} \in \operatorname{Fitt}_{O_{\chi}\left[\Gamma_{K}\right]}\left(A_{K_{\chi}}^{\chi}\right)$. Since $K_{\chi} / k$ satisfies the assumptions of Proposition 1, applying this proposition, for the character $\psi \in \widehat{\Gamma}_{K}$ as in the proposition we have $\psi\left(\theta_{K_{\chi} / k}^{\chi}\right)=\psi(x)$ for some $x \in \operatorname{Fitt}_{O_{\chi}\left[\Gamma_{K}\right]}\left(A_{K_{\chi}}^{\chi}\right)$. By the inclusion (5), we can write $x=\alpha \theta_{K_{\chi} / k}^{\chi}+y$ for some $\alpha \in O_{\chi}\left[\Gamma_{K}\right]$ and $y \in\left\langle\left\{v_{K_{\chi} / F}\left(\theta_{F / k}^{\chi}\right) \mid F \in \mathscr{M}_{K_{\chi} / k}, F \neq K_{\chi}\right\}\right\rangle_{O_{\chi}\left[\Gamma_{K}\right]}$. Since $\psi$ is faithful on $P_{\mathfrak{l}_{i}}$ for all $i$, we have $\psi(y)=0$, and therefore

$$
\psi\left(\theta_{K_{\chi} / k}^{\chi}\right)=\psi(x)=\psi(\alpha) \psi\left(\theta_{K_{\chi} / k}^{\chi}\right) .
$$

Since $\psi\left(\theta_{K_{\chi} / k}^{\chi}\right)=L\left(0,(\chi \psi)^{-1}\right) \neq 0$, we obtain $\psi(\alpha)=1$. This implies that $\alpha$ is a unit of the local ring $O_{\chi}\left[\Gamma_{K}\right]$. Note that $y \in \operatorname{Fitt}_{O_{\chi}\left[\Gamma_{K}\right]}\left(A_{K_{\chi}}^{\chi}\right)$ as we showed in the previous paragraph. We conclude that $\theta_{K_{\chi} / k}^{\chi}=\alpha^{-1}(x-y) \in \operatorname{Fitt}_{O_{\chi}\left[\Gamma_{K}\right]}\left(A_{K_{\chi}}^{\chi}\right)$. This completes the proof of the inclusion (6).

Proof of Theorem 3.5 in [3]. Let $K / \mathbf{Q}$ be an abelian extension satisfying the same conditions as that of Theorem 3.5. Namely, we assume that $K / \mathbf{Q}$ satisfies the condition (A) (see page 554 in [3]), and that $p$ is tamely ramified in $K$. We have to show (4) for all odd characters $\chi \in \widehat{\Delta}_{K}$.

For an odd character $\chi$ such that $p$ is ramified in $K_{(\Delta), \chi} / \mathbf{Q}$, the argument explained in the 4th paragraph on page 565 in [3] (in the proof of Theorem 3.5) shows that the equality (4) holds.

For an odd character $\chi$ such that $p$ is unramified in $K_{(\Delta), \chi} / \mathbf{Q}$, we have one-sided inclusion Fitt $O_{\chi}\left[\Gamma_{K}\right]\left(A_{K_{\chi}}^{\chi}\right) \subset\left(\Theta_{K_{\chi}} \otimes \mathbf{Z}_{p}\right)^{\chi}$ as we explained in the 3rd paragraph on page 565 in [3]. Since $\mu_{p} \not \subset K_{\chi}$ in this case, we can apply Proposition 1 to the abelian extension $K_{\chi} / \mathbf{Q}$ and obtain the other inclusion, using the same argument as the proof of Theorem 3.6 we just gave above.

Proof of Theorem 0.6 in [2]. Lemma 6.3 in [2] was used only to prove Theorems 0.4 and 0.6 in [2] by the first named author. We gave a correct proof of Theorem 0.4 above. Theorem 0.6 in [2] follows from Theorem 3.5 in [3] (as a special case). So both Theorems 0.4 and 0.6 in [2] are now proved. 
Remark The proof given in this correction was obtained in 2012. We explained this proof to several people at that time. But since we were asked similar questions several times, we have decided to publish this correction.

\section{References}

1. Kurihara, M.: On the ideal class groups of the maximal real subfields of number fields with all roots of unity. J. Eur. Math. Soc. 1, 35-49 (1999)

2. Kurihara, M.: Iwasawa theory and Fitting ideals. J. Reine Angew Math. 561, 39-86 (2003)

3. Kurihara, M., Miura, T.: Stickelberger ideals and Fitting ideals of class groups for abelian number fields. Math. Ann. 350, 549-575 (2011)

Publisher's Note Springer Nature remains neutral with regard to jurisdictional claims in published maps and institutional affiliations. 